\title{
Research on Metal Elastic - Plastic Deformation
}

\author{
Chao Zhang \\ Department of Mechanical Engineering, Shanghai University of Engineering Science, \\ Shanghai, China
}

\begin{abstract}
This paper establishes a finite element model to analyze the effect of different loading modes and technical parameters on quality of profile bending molding. This paper firstly gives a short introduction of the technique, basic principles and loading modes of profile stretch molding. The process of aluminum stretch molding and the snapping back phenomenon are then simulated by the finite element model. The effect of different loading modes and technical parameters on quality of profile bending molding is also analyzed. Simulations results indicate that different loading modes and technical parameters have significant influence on the quality of the profile bending molding.
\end{abstract}

\section{Keywords}

shaping profile bending; numerical Analysis; numerical simulation

\section{INTRODUCTION}

For the research on technology of profile stretch bending, the method of the experimental and numerical simulation analysis is the main development trend. By using the numerical model of finite element analysis software to establish the bending process, the performance parameters of the material obtained by uniaxial tension, biaxial tensile test are input finite element model, after the calculation and analysis, the influence of model parameters on the response parameters is calculated. Numerical simulation method can be used for studying various complex section profile, complex loading process, complex contact problem of stretch bending forming, and the current computer technology and finite element simulation technology is developing rapidly, the hardware and software of low cost and fast calculation speed, can greatly reduce the development cost and cycle of development of products. The disadvantages of numerical simulation of stretch bending in the profile bending are: the numerical simulation software is rarel, related domestic software information is rare. So it must be combined with the experimental analysis on the specific profile stretch bending.

\section{(1) Theory}

Elastic-plastic theory is based on mathematical relations to characterize the response of elastoplastic materials. There are three essential elements in the elastoplastic theory, ie. yield criteria, flow criteria and reinforcement criteria. The yield criterion relates the stress state to the occurrence of the material yield. The flow criterion relates the material stress state to the six increments corresponding to the plastic strain in the presence of incremental plastic flow. The reinforcement criterion describes how the strain-corrected yield criterion can be used after the material exceeds the initial yield.

(2) Yield criterion

The yield criterion determines the stress level at the start of yielding. For multi-component stresses, Yield is a function of each component, ie. $\mathrm{f}(\{\sigma\})$. Yield stress can also be understood as equivalent stress $\sigma_{e}$.

$$
\sigma_{e}=f(\{\sigma\})
$$

$\{\sigma\}---$ Stress vector

When the equivalent stress is equal to the material yield stress,

$$
f(\{\sigma\})=\sigma_{y}
$$

The material will produce a plastic strain. If $\sigma_{e}<\sigma_{y}$, the material is elastic and the stress varies according to the elastic stress-strain relationship.

If the equivalent stress is to be calculated in excess of the yield stress condition, shaping must be considered. The yield criterion also changes with the strengthening criterion. Take these factors into account (1-1). The yield function can be changed as follows.

$$
F(\{\sigma\}, \kappa,\{\alpha\})=0
$$

$\kappa$ - Plastic power; $\{\alpha\}$ - Yielding center moving stress vector $\kappa$ and $\{\alpha\}$ are state variables within the system. The plastic power is the sum of the plastic power in the loading process.

$$
\kappa=\int\{\sigma\}^{T}[M]\left\{d \varepsilon^{p l}\right\}
$$

In the formula,

$$
[M]=\left[\begin{array}{llllll}
1 & 0 & 0 & 0 & 0 & 0 \\
0 & 1 & 0 & 0 & 0 & 0 \\
0 & 0 & 1 & 0 & 0 & 0 \\
0 & 0 & 0 & 2 & 0 & 0 \\
0 & 0 & 0 & 0 & 2 & 0 \\
0 & 0 & 0 & 0 & 0 & 2
\end{array}\right]
$$

The displacement parameter of the yield surface is related to the loading history and is determined by the following equation.

$$
\{\alpha\}=\int C\left\{d \varepsilon^{p l}\right\}
$$


In the formula,

C- Material constants; $\{\alpha\}$-Back stress

(3) Flow criteria

The flow criterion describes the direction of plastic strain in the event of yielding. In other words, the flow criterion defines how a single plastic strain component develops with yield. The flow criterion determines the direction of the plastic strain and is determined by the following equation.

$$
\left\{d \varepsilon^{p l}\right\}=\lambda\left\{\frac{\partial Q}{\partial \sigma}\right\}
$$

In the formula,

\section{$\lambda$ - Plastic multiplier, Determine the plastic strain;}

Q- Plastic potential function, Determine the direction of plastic strain.

If the plastic potential function is equal to the yield stress function, the flow criterion is called the associated flow. In the associated flow, the plastic flow direction is the same as the outer normal direction of the yield surface. Associated flows are generally applicable to metals with extensibility. On the contrary, $Q \neq F$, Flows are unrelated flows. Non-associated flows are applied to soil or granular materials.

(4) Reinforcement criteria

The reinforcement criterion describes how the initial yield criterion develops with increasing plastic strain in order to establish the subsequent yield conditions. In the ANSYS program, you can use three reinforcement criteria: Isotropic strengthening, follow-up strengthening and mixing strengthening.

\section{BASIC PRINCIPLEAND TECHNOLOGY OF PROFILE STRETCH BENDING PROCESS}

\subsection{Basic principle of stretch bending}

The basic principle of stretch bending is adding tangential stress at the same time. So in the middle of bending, the stress distribution in the blank section tends to uniform, in order to reduce springback, improve the forming accuracy. Blank section stress is shown in figure 1 .

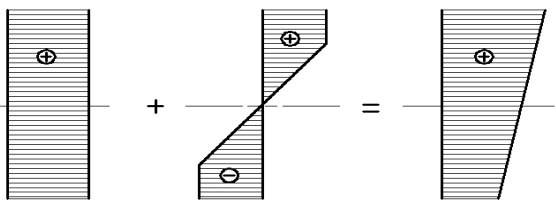

Fig. 1 The sketch of stress profile

As shown in Figure 2, profile is fixed in the chuck, and is added a bending moment to make it stick on the die; and through the tension, so that the stress distribution in the section tends to be uniform, and this method is applicable to extrusion (or bending) profiles of the frame type flange parts, pipe parts.
While bending tangential force is added on the blank with the inner pressure and lateral tension. On this basis the result is: original for lateral tension is to continue loading; for the inner original compression, a compression becomes tension, undergoing a process of unloading and reverse loading. In order to reflect the loading condition of stress strain relationship is reasonable, obviously the influence of elastic deformation can not be ignored.

\subsection{Research of stretch bending process loading}

At present two schemes are mainly used in production:one stretch bending and two stretch bending mode. One stretch bending tensile includes bending firstly then stretch bending and tensile bending finally and tension firstly then supplyment tension of these three kinds of loading mode. In production, it is mainly used to pull, then bend, finally add supplyment tension. The new quenching blank installed in the machine, to exceed the yield limit (stretching about $0.8 \sim 1 \%$ ), is made straight which can reduce the inner edge wrinkling trend in the bending process of thin-walled profile. Then to keep tension constant, add bending moment, the bending profile is contact on the die. Finally, to increase tension, to pull again, so that the inner fiber profiles of minimum curvature radius profiles is to tensile yield limit. There is still a small rebound in parts after unloading, when necessary, the size of springback shoud be fixed in die.

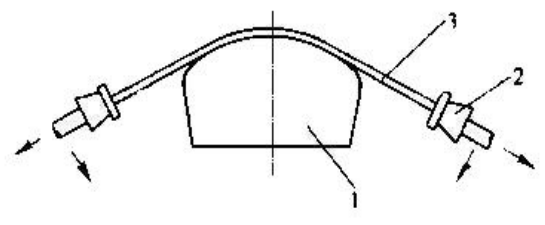

Fig. 2 The sketch of stretch bending

When the bending radius the parts is small and bend angle is larger, the effect of a stretch bending is poor. After bending supplyment tension can not completely eliminate inner stress, so the two stretch bending is adopted. Process of two stretch bending is: annealing pre tension -- Bending -- quenching -- two stretch bending under new quenching state. First annealing pre tension and bending do not include adding pull. As a result of small deformation of second bending, the inner compressive stress is easy because subsequent supplement tension is to be eliminated, so the parts is attached to die, the springback is small, the mold can not be repaired. Practice has proved: springback of parts is very small, mostly parts can be directly delivered. In this paper, mainly research is studied on application of the stretch bending forming.

\section{EFFECT OF DIFFERENT LOADING MODE AND PROCESS PARAMETERS ON PROFILE STRETCH BENDING FORMING 3.1 Effect of different bending modes on stretch bending of profile}

According to the modes and orders of the different loading, one stretch bending mode with three ways is commonly used. In this paper the three kinds of methods are studied with the finite element software MSC.MARC. Figure 3 shows: the T section profile edges bent in three bending process and the relationship of the total elongation and unloading rate. 
Three broken lines in Figure 3 show: with the increase in total elongation, springback decreases. When the amount of pre stretching and total elongation are same, the third kind of bending method can significantly reduce the springback. In third kind of bending, when the amount of the pre tension is invariant, the effect of total elongation on springback is more significant. In addition, in order to lighten the effect of friction, reduce the possibility of wrinkling and eliminate the possibility of initial deformation profiles, the third method is more superior than the first method. From the residual stress, the third kind of method has advantages over the second method. Therefore, the third kind of bending method is widely used in production.

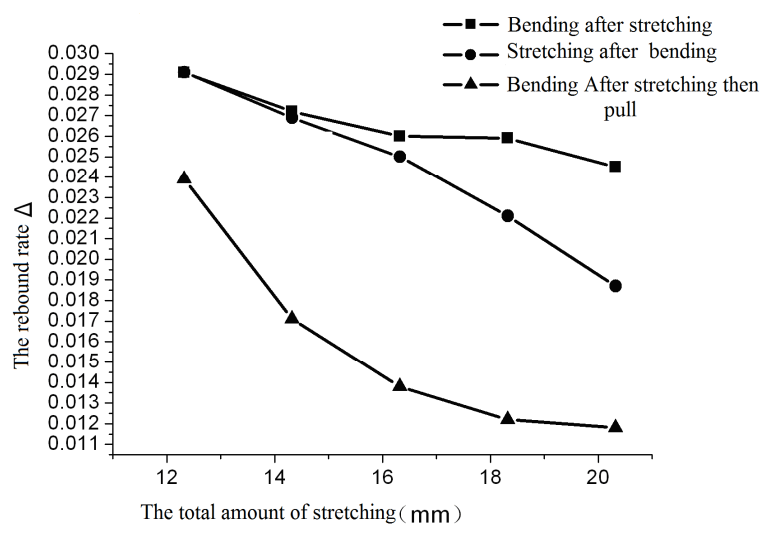

Fig. 3 The relationship of the three ways of strech aluminum bending and the rebound rate

\subsection{Effect of die radius on the springback of stretch bending of profile}

Be based on the finite element model, to keep the circular arc length size die face unchanged and chang the radius of curvature mould, $500 \mathrm{~mm}, 600 \mathrm{~mm}, 750 \mathrm{~mm}, 800 \mathrm{~mm}, 1000 \mathrm{~mm}$, therefore, the corresponding arc angle are $60^{\circ}, 50^{\circ}, 40^{\circ}, 37.5^{\circ}, 30^{\circ}$. Then, adjusting parameter of profile bending in the boundary condition of corresponding while pre stretching amount and supplemeng

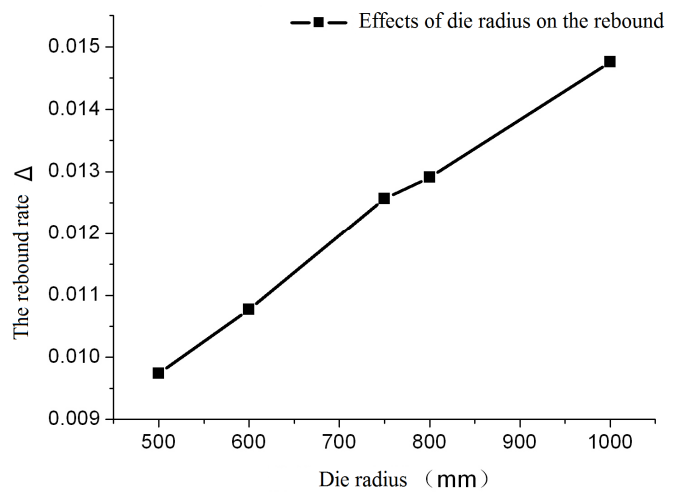

Fig.4 The relationship of the Die radius of curvature and rebound

stretching amount keep up the same. In this way,through a total of 5 times of simulation, the die radius have different effect on the rebound rate of profile stretch bending. It can be seen from Figure 4 , the die radius of curvature is more big, the springback after unloading rate is more high. Due to the bending part in production are mostly of the variable curvature, the research on this issue will be conducive to mold engineers repairing model accurately.

\section{CONCLUSIONS}

The results can be seen. For stretch bending of the T section, the first stretch bending then supplymeng strentch is an excellent bending mode. The amount of pre stretching have a certain effect on the forming quality of the parts. If pre stretching is insufficient, the phenomenon of wrinkling will occur easily. If the total elongation fixed, when the aluminum profile received more pre stretching amount, rebound has a tendency to increase. When the prestretching is fixed, the springback of $\mathrm{T}$ aluminum profile will decrease with the increase of supplyment strench amount. Research results showed that, in the $\mathrm{T}$ section of stretch bending of aluminum, with increasing the die radius, springback increases, friction has little effect on the springback.

\section{REFERENCES}

[1] Zhai Ping, principle and process of sheet metal forming, the Northwestern Polytechnical University press, 2010, 145-154

[2] Xie Lansheng, Hu Hao, the springback finite-element analysis profile bending, aviation precision manufacturing technology, 2012 , Vol.40 issue 5, 34-36

[3] Jin Miao, Zhou Xianbin, etc., the large size of closed section aluminum profile stretch bending process research, Journal of Plasticity Engineering, 2011(11), Vol.10 issue 5, 46-49

[4] Li Hongwei, the big section forming process, scientific technology, 2012 (2), 29-31

[5] Frode Paulsen, Torgeir Welo, A design method for prediction of dimensions of rectangular hollow sections formed in stretch bending, Journal of Materials Processing Technology, 128(2009)48-66

[6] Edmundo Corona , A simple analysis for bend-stretch forming of aluminum extrusions, International Journal of Mechanical Sciences ,46 (2010) 433-448

[7] Zhai Ping, principle and process of sheet metal forming, the Northwestern Polytechnical University press,2010, 145-154

[8] Xie Lansheng, Hu Hao, the springback finite-element analysis profile bending, aviation precision manufacturing technology, 2012 , Vol.40 issue 5, 34-36

[9] Jin Miao, Zhou Xianbin, etc., the large size of closed section aluminum profile stretch bending process research, Journal of Plasticity Engineering, 2011(11), Vol.10 issue 5, 46-49

[10] Li Hongwei, the big section forming process, scientific technology, 2012 (2), 29-31

[11]Frode Paulsen, Torgeir Welo, A design method for prediction of dimensions of rectangular hollow sections formed in stretch bending, Journal of Materials Processing Technology, 128(2009)48-66 